\title{
Instrumentos utilizados para avaliaçáo do desenvolvimento de recém-nascidos pré-termo no Brasil: revisão da literatura
}

\author{
Solange Raydan Rocha ${ }^{a}$, Lílian de Fátima Dornelas ${ }^{b}$, Lívia de Castro Magalhães ${ }^{c}$ \\ ${ }^{a}$ Terapeuta ocupacional, Universidade Federal de Minas Gerais - UFMG, Belo Horizonte, MG, Brasil \\ 'Fisioterapeuta, Doutoranda em Ciências da Reabilitação, Universidade Federal de Minas Gerais - UFMG, \\ Belo Horizonte, MG, Brasil \\ 'Doutora, Professora do Departamento de Terapia Ocupacional e do Programa de Pós-graduação em Ciência da \\ Reabilitação da Escola de Educação Física, Fisioterapia, Terapia Ocupacional - EEFFTO, \\ Universidade Federal de Minas Gerais - UFMG, Belo Horizonte, MG, Brasil
}

\begin{abstract}
Resumo: Os avanços tecnológicos na assistência ao neonato têm levado ao aumento da sobrevida dos recém-nascidos pré-termo. Esse aumento tem gerado preocupação quanto ao desenvolvimento desses bebês e provocado interesse dos pesquisadores em investigar o desenvolvimento neuropsicomotor de crianças que nasceram prematuramente. Revisar a literatura brasileira para identificar os principais instrumentos utilizados para avaliar o desenvolvimento de crianças nascidas pré-termo nos primeiro três anos de vida foi o objetivo deste trabalho. Foi realizado levantamento bibliográfico, nos anos de 1999 a 2011, nas bases de dados Lilacs e Scielo. Foram identificados 11 instrumentos de avaliação do desenvolvimento, sendo que, dos 24 artigos selecionados para a revisão, a maioria $(11 ; 45,83 \%)$ utilizou as escalas Alberta Infant Motor Scale (AIMS) $(6 ; 54,5 \%)$ e o teste de triagem do desenvolvimento Denver II (TDD-II) (5; 45,5\%). Há predomínio do uso de instrumentos referenciados em normas, criados em outros países, sem validação para a criança brasileira. A revisão indica a necessidade de mais pesquisas voltadas para a tradução e validação tanto dos testes importados como dos instrumentos desenvolvidos no Brasil.
\end{abstract}

Palavras-chave: Prematuridade, Desenvolvimento Infantil, Avaliação.

\section{Assessment tools utilized for the evaluation of preterm neonates in Brazil: literature review}

\begin{abstract}
Introduction: Technological advances in neonatal care have led to increased survival of preterm newborns. This increase has raised concern about the development of these babies and generated interest among researchers to investigate the neuropsychomotor development of children born prematurely. Objective: To review the Brazilian literature to identify the main instruments used to evaluate the development of children born preterm in the first three years of life. Methodology: A literature search, from 1999 to 2011, was conducted in the Lilacs and Scielo databases. Results: Eleven developmental assessments were identified, and among the 24 articles selected for this review, most of them $(11,45.83 \%)$ used the Alberta Infant Motor Scale (AIMS) (6, 54.5\%) and the Developmental Screening test Denver II (DDR II) (5, 45.5\%). Conclusion: There is predominance of the use of norm-referenced instruments created in other countries, with no validation studies for the Brazilian children. This review indicates a need for more research focused on the translation and validation of international assessment tools as well as instruments developed in Brazil.
\end{abstract}

Keywords: Prematurity, Child Development, Assessment.

Autor para correspondência: Lívia de Castro Magalhães, Escola de Educação Física, Fisioterapia e Terapia Ocupacional, Universidade Federal de Minas Gerais, Av. Presidente Antônio Carlos, 6627, Campus Pampulha, CEP 31270-901, Belo Horzionte, MG, Brasil, e-mail: liviam@gcsnet.com.br

Recebido em 28/6/2012; $1^{\text {a }}$ Revisão em 8/8/2012; Aceito em 16/8/2012. 


\section{Introdução}

Bebês pré-termo são mais vulneráveis ao atraso no desenvolvimento, pois a prematuridade interrompe os processos de maturação do cérebro, o que pode levar a alteraçóes anatômicas e estruturais, podendo resultar em incapacidades funcionais, cognitivas e comportamentais (ZOMIGNANI; ZAMBELLI; ANTÔNIO, 2009).

Esses bebês geralmente apresentam intercorrências clínicas (ex.: hemorragia intra e periventricular, hipóxia neonatal, baixo peso ao nascer, imaturidade pulmonar, crises convulsivas, infecçoes, entre outras) e devido ao longo período de internaçáo, com intervençôes e manipulações muitas vezes invasivas e dolorosas, encontram-se privados de vários estímulos do ambiente e do contato com a máe, importantes para o desenvolvimento neuropsicomotor (DNPM) (AYACHE; MARIANI NETO, 2003). Como resultado de uma combinação de fatores, é fato que o desenvolvimento do bebê pré-termo, nos primeiros meses de vida, ocorre de forma diferente, pois apresenta imaturidade funcional que leva a experiências diferenciadas de movimento, podendo influenciar o desenvolvimento motor (VIEIRA; RIBEIRO; FORMIGA, 2009).

Tendo em vista a prematuridade como fator de risco para o desenvolvimento infantil, faz-se necessário o acompanhamento dos bebês nascidos prematuramente por meio de avaliaçóes do desenvolvimento neuropsicomotor (DNPM). Para fazer a detecção eficiente do atraso no desenvolvimento neuropsicomotor (ADNPM) em crianças nascidas pré-termo há necessidade da utilização de observaçóes criteriosas, sendo recomendado o uso de testes padronizados para avaliação do desenvolvimento (FORMIGA; LINHARES, 2009; SILVA et al., 2011).

Testes padronizados são aqueles que têm procedimentos uniformes para coleta de dados e interpretação dos resultados (RICHARDSON, 2001). O teste deve ser bem formulado, com normas claras de aplicação e com resultados/escores quantificados. Todo instrumento de medida deve apresentar índices de validade e confiabilidade adequados à população na qual será utilizado, para garantir mensuração adequada na prática clínica, com nível máximo de consistência e acuidade (ANASTASI; URBINA, 2000).

$\mathrm{Na}$ literatura internacional encontramos referências de vários testes motores, para crianças de diferentes idades, que são muito utilizados na clínica e na pesquisa. No Brasil, no entanto, há escassez de instrumentos padronizados e confiáveis para avaliar habilidades nos primeiros anos de vida de nossas crianças (SANTOS; ARAÚJO; PORTO, 2008). A escassez de testes motores padronizados e com normas de desempenho por idade para crianças brasileiras dificulta tanto a identificação correta de crianças que poderiam se beneficiar de programas de estimulação como o desenvolvimento de pesquisas.

É importante que os profissionais tenham conhecimento dos recursos disponíveis, dentro e fora do país, para poder fazer escolhas apropriadas e aplicar corretamente os instrumentos, de forma a garantir maior eficácia na detecçáo de problemas e na avaliação do desfecho de programas de intervenção (AYACHE; MARIANI NETO, 2003).

Como alguns testes citados na literatura internacional vêm sendo utilizados por pesquisadores brasileiros, é importante levantar quais são utilizados e com qual finalidade. O presente estudo teve como objetivo realizar revisão de literatura, visando localizar, identificar e analisar estudos originais publicados no Brasil que incluíram utilização de testes padronizados para avaliação do desenvolvimento motor de crianças de 0 a 3 anos nascidas prematuramente.

\section{Metodologia}

A busca dos artigos foi realizada nas bases de dados Lilacs e Scielo, utilizando-se as seguintes palavras-chave, em português: pré-termo; avaliação; desenvolvimento; acompanhamento do desenvolvimento; deficiência; prematuridade; desenvolvimento motor.

\subsection{Critérios de inclusão}

Artigos originais publicados no Brasil nos anos de 1999 a 2011, que se referiam à utilização de instrumentação padronizada para avaliação do desenvolvimento da criança nascida pré-termo de 0 a 3 anos de idade. Foram considerados como instrumentos padronizados os testes que têm manual com instruçóes detalhadas de como administrar, pontuar e interpretar os escores, assim como tabelas com normas de desempenho por idade.

\subsection{Critérios de exclusão}

Artigos que não utilizavam instrumentos padronizados, artigos de revisão e estudos de caso.

\section{Resultados}

Como ilustrado na Figura 1, foram selecionados 24 artigos para análise crítica do presente estudo. 


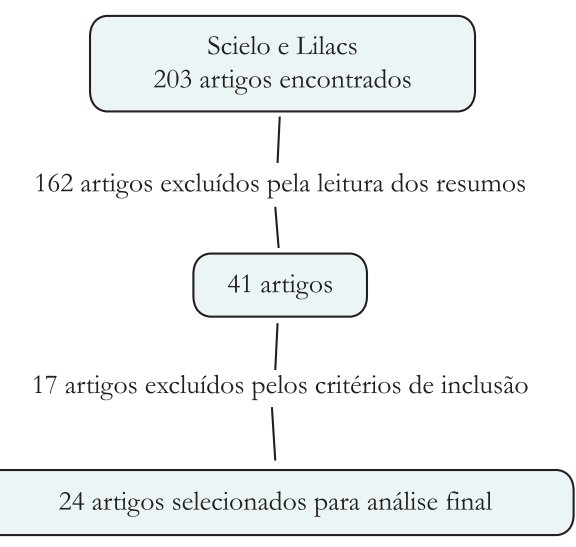

Figura 1. Estágios da busca e localização de artigos para revisão.

Foram identificados 11 instrumentos de avaliação do desenvolvimento, que são descritos brevemente no quadro 1 . Os testes mais utilizados nos estudos acerca do desenvolvimento da criança pré-termo no Brasil foram Alberta Infant Motor Scale (AIMS) (ZANINI et al., 2002; MANCINI; CARVALHO; GONTIJO, 2002; FORMIGA; PEDRAZZANI; TUDELLA, 2004; RESTIFFE; GHERPELLI, 2006; ALMEIDA et al., 2008; MANACERO; NUNES, 2008) e o teste de triagem do desenvolvimento Denver II (TDD-II) (MAGALHÃES et al., 1999, 2011; RESEGUE; PUCCINI; SILVA, 2008; KRELING; BRITO; MATSUO, 2006; CHERMONT et al., 2005). Para nomes e referência completa dos testes citados veja Apêndice I.

A escala AIMS foi utilizada com o objetivo de verificar a necessidade de correçáo da idade cronológica (ZANINI et al., 2002; MANCINI; CARVALHO; GONTIJO, 2002; RESTIFFE; GHERPELLI, 2006), identificar progresso em intervenção (FORMIGA; PEDRAZZANI; TUDELLA, 2004) e investigar a validade do instrumento (ALMEIDA et al., 2008; MANACERO; NUNES, 2008). Já o teste Denver II foi utilizado para identificar ADNPM (KRELING; BRITO; MATSUO, 2006), traçar o perfil das crianças (RESEGUE; PUCCINI; SILVA, 2008) e analisar a interferência de fatores de risco no desenvolvimento (CHERMONT et al., 2005). Ressaltou-se a necessidade de validar medidas de desenvolvimento para a população brasileira (MAGALHĀES et al., 1999, 2011).

Notou-se que todos os instrumentos encontrados foram desenvolvidos para população de outros países (quadro 1), com exceção da EDCC (LINHARES et al., 2003; GUIMARÃES et al., 2003), que foi criada e vem sendo validada para a população brasileira, e do PEDI (MANCINI et al.,
2002) que, embora criado nos EUA, foi traduzido e feita adaptação transcultural para a criança brasileira (MANCINI, 2005), com publicação de manual de instruçóes e normas de desempenho, em português.

Nos artigos estudados foram encontrados 12 estudos longitudinais (MAGALHĀES et al., 2011; RANIERO; TUDELLA; MATTOS, 2010; RESTIFFE; GHERTIFFE, 2006; KRELING; BRITO; MATSUO, 2006; LACERDA; MAGALHÁES, 2006; CARDOSO et al., 2004; GUIMARÁES et al., 2003; LINHARES et al., 2003; MANCINI et al., 2002; MANCINI; CARVALHO; GONTIJO, 2002; ZANINI et al., 2002; MAGALHĀES et al., 1999), oito transversais (MAGALHÁES et al., 2001; CHERMONT et al., 2005; ROSA NETO, 2002; BARBOSA et al., 2007; FRAGA et al., 2008; ALMEIDA et al., 2008; MANACERO; NUNES, 2008; GUIMARÃES et al., 2011), dois retrospectivos (RESEGUE; PUCCINI; SILVA, 2008; BONVICINE et al., 2004) e dois ensaios clínicos randomizados (FORMIGA; PEDRAZZANI; TUDELLA, 2004; BARRADAS et al., 2006).

Em todos os artigos foram utilizados um ou dois instrumentos para avaliação e/ou acompanhamento de crianças pré-termo. Dentre os estudos, quatro (ALMEIDA et al., 2008; LACERDA; MAGALHÃES, 2006; CARDOSO et al., 2004; MAGALHÃES et al., 1999) foram voltados para examinar qualidades psicométricas dos testes (ex: confiabilidade, validade de constructo, concorrente e preditiva) e o restante (20 estudos) teve foco específico na prematuridade, o que incluiu comparar grupos, verificando diferenças entre crianças nascidas pré-termo e a termo (ZANINI et al., 2002; MANCINI; CARVALHO; GONTIJO, 2002; MANCINI et al., 2002; BONVICINE et al., 2004; RANIERO; TUDELLA; MATTOS, 2010; GUIMARÁES et al., 2011), examinar a influência de fatores de risco para o desenvolvimento infantil (KRELING; BRITO; MATSUO, 2006; MANACERO; NUNES, 2008; RESEGUE; PUCCINI; SILVA, 2008; FRAGA et al., 2008; BARBOSA et al., 2007; CHERMONT et al., 2005; MAGALHÁES et al., 2001), comparar as características de desenvolvimento do grupo de risco com os dados normativos dos testes (MANACERO; NUNES, 2008; ROSA NETO, 2002; MAGALHÁES et al., 2001, 2011; CHERMONT et al., 2005; GUIMARÁES et al., 2003; LINHARES et al., 2003), verificar necessidade de correçấo da idade para acompanhamento das aquisiçóes motoras (RESTIFFE; GHERPELLI, 2006) e documentar efeitos de intervenção 


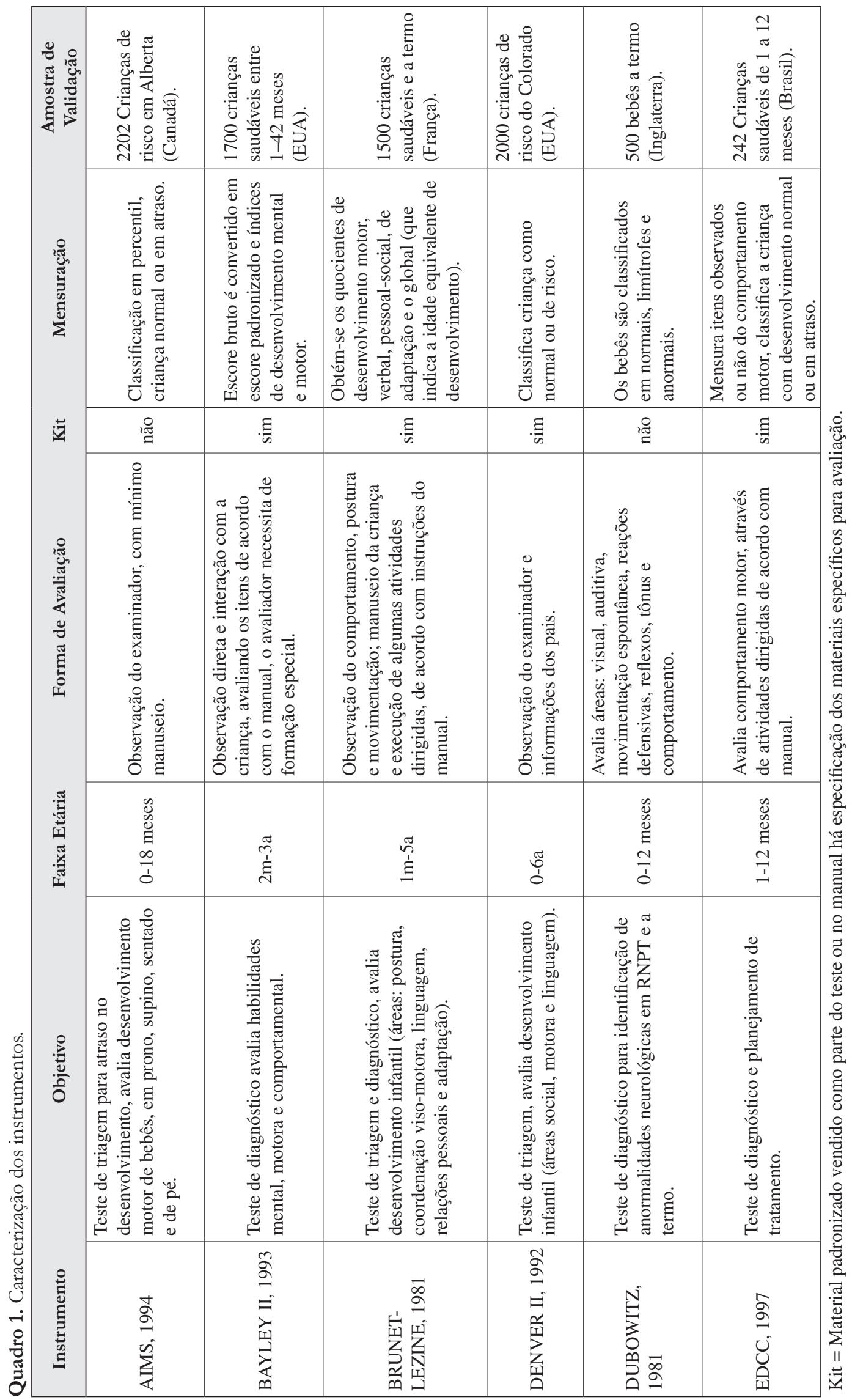


(BARRADAS et al., 2006; FORMIGA; PEDRAZZANI; TUDELLA, 2004).

Observou-se que a maior produção científica nessa área ficou a cargo dos fisioterapeutas, com 12 artigos publicados (50\%) (RANIERO; TUDELLA; MATTOS, 2010; MANACERO; NUNES, 2008; ALMEIDA et al., 2008; BARBOSA et al., 2007; RESTIFFE; GHERPELLI, 2006; KRELING; BRITO; MATSUO, 2006; BARRADAS et al., 2006; LACERDA; MAGALHÂES, 2006; BONVICINE et al., 2004; FORMIGA; PEDRAZZANI; TUDELLA, 2004; GUIMARÁES et al., 2003; ZANINI et al., 2002), seguidos pelos terapeutas ocupacionais com seis artigos (25\%) (MAGALHÂES et al., 1999, 2001; 2011; MANCINI; CARVALHO; GONTIJO, 2002; MANCINI et al., 2002; CARDOSO et al., 2004), os pediatras com três artigos (12,50\%) (CHERMONT et al., 2005; RESEGUE; PUCCINI; SILVA, 2008; GUIMARĀES et al., 2011), os psicólogos com dois artigos (8,33\%) (FRAGA et al., 2008; LINHARES et al., 2003) e os educadores físicos com um artigo (4,17\%) (ROSA NETO, 2002).

No Brasil, as publicaçôes científicas acerca da temática da avaliaçáo motora da criança nascida pré-termo se concentram na região Sudeste, especialmente nos estados de São Paulo (RANIERO; TUDELLA; MATTOS, 2010; RESEGUE; PUCCINI; SILVA, 2008; FRAGA et al., 2008; BARBOSA et al., 2007; RESTIFFE; GHERPELLI, 2006; BONVICINE et al., 2004; GUIMARÃES et al., 2003; LINHARES et al., 2003; ZANINI et al., 2002) e Minas Gerais (MAGALHÂES et al., 1999, 2001, 2011; MANCINI; CARVALHO; GONTIJO, 2002; MANCINI et al., 2002; CARDOSO et al., 2004; LACERDA; MAGALHÃES, 2006; ALMEIDA et al., 2008), com 17 artigos publicados (70,83\%), seguidos do Sul (CHERMONT et al., 2005; KRELING; BRITO; MATSUO, 2006; ROSA NETO, 2002; MANACERO; NUNES, 2008) com quatro artigos publicados, Nordeste (BARRADAS et al., 2006; GUIMARÁES et al., 2011) com dois artigos, e Centro-Oeste (FORMIGA; PEDRAZZANI; TUDELLA, 2004) com um artigo.

\section{Discussão}

Todos os instrumentos identificados neste estudo foram utilizados para avaliar o desenvolvimento motor de crianças prematuras de 0 a 3 anos de idade. Cada instrumento possui objetivo específico, sendo alguns adequados para triagem de ADNPM e outros para diagnóstico de lesóes neurológicas. Portanto, é necessária a escolha correta do instrumento, pois cada instrumento de avaliação tem uma finalidade específica, de acordo com os objetivos definidos para coleta de dados e implementação da detecção ou intervenção a ser realizada.

É de grande importância clínica que seja utilizado um instrumento com normas e critérios de referência, para mensuração confiável de dados clínicos relevantes. Observa-se, com base nos estudos analisados, o pequeno número de instrumentos com validaçáo e padronização para a população brasileira, sendo essencial investir na padronização dos instrumentos existentes em nosso país ou publicados no exterior, para permitir a triagem ou identificação confiável em atraso no desenvolvimento psicomotor. Os instrumentos utilizados tanto na pesquisa e, especialmente, na clínica, devem ser sensíveis para detectar sinais indicativos de atrasos em crianças brasileiras, de diferentes regióes do país e níveis sociais, para possibilitar orientação e intervenção o mais precocemente possível. O uso de testes importados pode comprometer a avaliação $\mathrm{da}$ criança de risco, por não apresentarem normas de desempenho padronizadas para nossa populaçáo e possuírem manuais que ainda náo foram traduzidos, dificultando ainda mais sua utilização correta.

Dentre os instrumentos citados nas pesquisas, os de maior utilização foram a AIMS (PIPPER; DARRAH, 1994) e o TDD-II (FRANKENBURG et al., 1992). Ambos são testes para triagem de atraso no desenvolvimento, de fácil aplicação, custo relativamente baixo, que possuem manual de instrução detalhado e não necessitam de grande treinamento para aplicação. A facilidade de uso e o custo possivelmente influenciam sua utilização, não só em nosso meio, mas também internacionalmente, pois são testes bastante citados em estudos em diferentes países. Além disso, são instrumentos com confiabilidade e validade bem documentadas, que vêm sendo usados na clínica e podem ser utilizados em acompanhamentos longitudinais e em programas de follow-up. O teste TDD-II oferece ainda a vantagem de ser uma avaliaçáo do desenvolvimento global e apresentar uma faixa etária de maior abrangência, o que contribui para seu maior uso.

É importante ressaltar, no entanto, que embora esses dois testes já tenham tido suas propriedades psicométricas examinadas em alguns estudos brasileiros, ainda não existem traduçóes oficiais/ autorizadas nem normas de desempenho para a população brasileira, o que deve ser feito antes de se recomendar sua utilização para decisóes clínicas.

Por outro lado, a EDCC (PINTO; VILANOVA; VIEIRA, 1997), que é a única escala criada para 
a população brasileira, ainda é pouco utilizada e conhecida no meio clínico, talvez devido à faixa etária restrita de utilização. Seria interessante conduzir mais estudos com essa escala, ou mesmo que o grupo de autores desse continuidade ao trabalho, avançando pelo menos até os três anos de idade.

O teste PEDI (HALEY et al., 1992) foi desenvolvido com crianças norte-americanas, mas foi traduzido, adaptado e conta com dados normativos para nossa população (MANCINI, 2005). Esse teste é usado para avaliar o desempenho da criança nas tarefas de vida diária, identificando atrasos ou déficits funcionais, sendo muito útil em programas de intervençáo. É um recurso que vem sendo muito utilizado na prática clinica em nosso país por apresentar evidências de boa utilidade clínica e abranger uma faixa etária ampla de crianças, sendo útil para planejar programas voltados para melhoria do desempenho funcional de crianças de 6 meses a 7 anos e 6 meses, além de contar com vários trabalhos publicados no país, que dão suporte a sua utilização.

O teste NAPI foi o único instrumento utilizado exclusivamente para avaliação do desenvolvimento neurocomportamental e de sinais de alerta no RNPT. É utilizado em bebês de 32 a 40 semanas de idade pós-concepcional, para avaliar o desempenho neurológico e comportamental de RNPT, acompanhar efeitos de intervenção e identificar atrasos no desenvolvimento do bebê. Esse instrumento tem ainda pouca utilização clínica no Brasil, tendo sido encontrado apenas um artigo com sua utilização (BARBOSA et al., 2007).

O teste MAI (CHANDLER; ANDREWS; SWANSON, 1980) foi o teste mais utilizado para detecção de distúrbios neuromotores. É um instrumento que tem vários estudos de validade, confiabilidade e bom valor preditivo para problemas neurológicos (CARDOSO et al., 2004; LACERDA; MAGALHÃES, 2006), mas é um teste mais antigo, que enfatiza a avaliaçáo de reflexos primitivos, o que é pouco compatível com os modelos atuais de intervenção. Segundo Lacerda e Magalhães (2006), alguns itens do teste devem ser revisados para aumentar a validade e confiabilidade do instrumento.

Além dos testes citados, encontramos também referência a outros instrumentos, que não foram incluídos no estudo por apresentarem apenas padronização de instruções, mas que não apresentam dados normativos. Dentre estes, encontramos dois artigos que citam utilização do Inventário Portage Operacionalizado - IPO (WILLIAMS; AIELLO, 2001), que é um roteiro para planejamento de programa de estimulação, para crianças de 0 a 6 anos. Outro teste citado em um artigo foi o Exame
Neuropsicomotor de Gesell-Gesell Developmental Schedules (GESELL; AMATRUDA, 1945), para crianças de 0 a 5 anos, mas que náo tem normas de desempenho por idade (WILLIAMS; AIELLO, 2001; TECKLIN, 2002).

Esta revisão indica que a atual produção científica do país com utilização de testes padronizados para avaliaçáo do desenvolvimento motor de crianças nascidas pré-termo se concentra na região Sudeste, com predomínio de atividades desenvolvidas em niversidades públicas, vinculadas a programas de follow-up de crianças de risco, o que sugere maior incentivo a projetos de pesquisa nessas instituiçôes. A maioria desses projetos foi desenvolvida por fisioterapeutas, seguidos de terapeutas ocupacionais, que talvez sejam os profissionais mais presentes nas equipes de acompanhamento do desenvolvimento de RNPT. Os fisioterapeutas, além da tradição no estudo do desempenho motor, são profissionais mais numerosos e com maior envolvimento com programas de pós-graduação.

Observa-se que um número considerável de testes motores importados vem sendo usado em pesquisa no nosso país, gerando uma variedade de estudos, de intervençáo e descrição ou comparação do desempenho motor entre crianças nascidas pré-termo e a termo. Encontramos apenas um teste criado no Brasil, o que aponta para dificuldades de investimento no longo processo de criação de instrumentos para avaliação do desenvolvimento infantil. É importante salientar que ainda são poucos os estudos voltados para o exame da validade e utilidade dos instrumentos para uso com crianças brasileiras. Aspectos culturais e socioeconômicos relacionados à validade dos instrumentos são negligenciados, o que é preocupante, considerando que a maioria dos testes foi criada em países de primeiro mundo, onde as famílias e crianças têm acesso a diferentes recursos, que podem influenciar o desempenho motor, resultando em possível inadequação das normas originais de desempenho, o que precisa ser examinado.

Devido à importância do acompanhamento do recém-nascido de risco, em programas de follow-up, é importante o uso do instrumento correto, com normas e/ou critérios de referência adequados, para avaliar, diagnosticar e planejar o tratamento de crianças brasileiras de diferentes níveis sociais. Instrumentos que permitem acompanhamento longitudinal possibilitam melhor aplicação clínica, pois mostram a progressão de habilidades e favorecem programas de intervenção. Vários instrumentos já vêm sendo usados, sendo importante investir em estudos de validação. 


\section{Conclusão}

Há predomínio do uso de instrumentos importados sem validação para a criança brasileira. A revisão indica a necessidade de mais pesquisas voltadas para a tradução e validação tanto dos testes importados como dos instrumentos desenvolvidos no Brasil. $\mathrm{O}$ uso de instrumentos padronizados aumenta a confiabilidade e rigor na identificaçáo de atraso motor e acompanhamento da criança de risco, o que pode contribuir para avaliação e melhoria da prestação de serviços na área de estimulação do desenvolvimento infantil em nosso país, pois vai permitir a comparaçáo do desempenho não só de crianças, mas também de programas ao longo do tempo.

\section{Referências}

ALMEIDA, K. M. et al. Validade concorrente e confiabilidade da Alberta Infant Motor Scale em lactentes nascidos prematuros. Jornal de Pediatria, Rio de Janeiro, v. 84 , n. 5 , p. $442-448$, set./out. 2008 . http://dx.doi. org/10.2223/JPED.1836

ANASTASI, A.; URBINA, S. Testagem Psicológica. Porto Alegre: Artmed, 2000.

AYACHE, M. G.; MARIANI NETO, C. Consideraçôes sobre o desenvolvimento motor do prematuro. Temas sobre desenvolvimento, São Paulo, v. 12, n. 7, p. 5-9, nov./dez. 2003.

BARBOSA, V. C.; FORMIGA, C. K. M. R.; LINHARES, M. B. M. Avaliação das variáveis clínicas e neurocomportamentais de recém-nascidos pré-termo. Revista Brasileira de Fisioterapia, Sáo Carlos, v. 11, n. 4, p. 275-281, 2007. http://dx.doi.org/10.1590/ S1413-35552007000400006

BARRADAS, J. et al. A relação entre posicionamento do prematuro no Método Mãe-Cangurú e desenvolvimento neuropsicomotor precoce. Jornal de Pediatria, Rio de Janeiro, v. 82, n. 6, p. 475-480, 2006. http://dx.doi. org/10.1590/S0021-75572006000800014

BONVICINE, C. et al. Prematuridade como possível influenciador do controle da cabeça. Salusvita, Bauru, v. 23, n. 3, p. 489-500, 2004.

CARDOSO, A. A. et al. Validade preditiva do Movement Assessment of Infants para crianças pré-termo brasileiras. Arquivos de Neuro-Psiquiatria, São Paulo, v. 62, n. 4, p. 1052-1057, dez. 2004. http://dx.doi.org/10.1590/ S0004-282X2004000600022

CHANDLER, L. S.; ANDREWS, M. S.; SWANSON, M. W. Movement assessment of infants: A. Manual. Washington: University of Washington, 1980.

CHERMONT, A. G. et al. Avaliaçáo do desenvolvimento pela escala de DenverII, de recém-nascidos prematuros de baixo peso. Revista Paraense de Medicina, Belém, v. 19, n. 2, p. 59-66, 2005.
FORMIGA, C. K. M. R.; LINHARES, M. B. M. Avaliação do desenvolvimento inicial de crianças nascidas pré-termo. Revista da Escola de Enfermagem da USP, São Paulo, v. 43, n. 2, p. 472-480, jun. 2009. http://dx.doi. org/10.1590/S0080-62342009000200030

FORMIGA, C. K. M. R.; PEDRAZZANI, E. S.; TUDELLA, E. Desenvolvimento motor de lactentes pré-termo participantes de um programa de intervenção fisioterapêutica precoce. Revista Brasileira de Fisioterapia, São Carlos, v. 8, n. 3, p. 239-245, set./dez. 2004.

FRAGA, D. A. et al. Desenvolvimento de bebês prematuros relacionado a variáveis neonatais e maternas. Psicologia em Estudo, Maringá, v. 13, n. 2, p. 335-344, 2008. http:// dx.doi.org/10.1590/S1413-73722008000200016

FRANKENBURG, W. K. et al. DenverII Screening Manual. Denve: Denver Developmental Materials, Inc., 1992.

GESELL, A.; AMATRUDA, C. Diagnostico del desarrollo normal y anormal del nino: métodos clínicos y aplicaciones practicas. Buenos Aires: Medico Quirurgica, 1945.

GUIMARÃES, C. L. N. et al. Desenvolvimento motor avaliado pelo Test of Infant Motor Performance: comparação entre lactentes pré-termo e a termo. Revista Brasileira de Fisioterapia, Sáo Carlos, v. 15, n. 5, p. 357-362, 2011. http://dx.doi.org/10.1590/ S1413-35552011005000021

GUIMARÃES, E. L. et al. Estudo comparativo do desenvolvimento de recém-nato pré-termo aos quatro e seis meses de vida, segundo a escala "o desenvolvimento do comportamento da criança nos primeiros anos de vida". Fisioterapia em Movimento, Curitiba, v. 16, n. 4, p. 41-46, 2003.

HALEY, S. M. et al. Pediatric evaluation of disability inventory (PEDI): development, standardization and administration manual. version 1.0. Boston: PEDI Research Group. New England Medical Center Inc, 1992.

KRELING, K. C. A.; BRITO, A. S. J.; MATSUO, T. Fatores perinatais associados ao desenvolvimento neuropsicomotor de recém-nascidos de muito baixo peso. Pediatria, São Paulo, v. 28, n. 2, p. 98-108, 2006.

LACERDA, T. T. B.; MAGALHĀES, L. C. Análise da validade dos itens do Movement Assessment of Infants - MAI - para crianças pré-termo. Revista Brasileira Saúde Materno Infantil, Recife, v. 6, n. 3, p. 297-308, jul./dez. 2006. http://dx.doi.org/10.1590/ S1519-38292006000300006

LINHARES, M. B. M. et al. Desenvolvimento de bebês nascidos pré-termo no primeiro ano de vida. Paidéia, Ribeirão Preto, v. 13, n. 25, p. 59-72, jan./jun. 2003. http://dx.doi.org/10.1590/S0103-863X2003000200006

MANCINI, M. C. et al. Estudo do desenvolvimento da função motora aos 8 e 12 meses de idade em crianças nascidas pré-termo e a termo. Arquivos de NeuroPsiquiatria, São Paulo, v. 60, n. 4, p. 974-980, 2002a. http://dx.doi.org/10.1590/S0004-282X2002000600017

MANCINI, M. C.; CARVALHO, D. J.; GONTIJO, D. T. Os efeitos da correção da idade no desempenho 
motor grosso e fino de crianças pré-termo aos dois anos de idade. Temas sobre Desenvolvimento, São Paulo, v. 11, n. 64, p. 12-19, set./out. 2002b.

MANCINI, M. C. Inventário de avaliação pediátrica de incapacidade (PEDI): manual da versão brasileira adaptada. Belo Horizonte: UFMG, 2005.

MANACERO, S.; NUNES, M. L. Avaliação do desempenho motor de prematuros nos primeiros meses de vida na Escala Motora Infantil de Alberta (AIMS). Jornal de Pediatria, Rio de Janeiro, v. 84, n. 1, p. 53-59, jan./fev. 2008. http://dx.doi.org/10.1590/ S0021-75572008000100010

MAGALHÃES, L. C. et al. Influência de fatores de risco biológico nos escores de um teste para detecção de paralisia cerebral em crianças pré-termo. Temas sobre Desenvolvimento, São Paulo, v. 10, n. 5, p. 5-12, set./ dez. 2001.

MAGAlHÃES, L. C. et al. Análise do desempenho de crianças pré-termo no Teste de Desenvolvimento de Denver nas idades de 12, 18 e 24 meses. Pediatria, São Paulo, v. 21, n. 4, p. 330-339, 1999.

MAGALHÂES L. C. et al. Desempenho de crianças pré-termo com muito baixo peso e extremo baixo peso segundo o teste Denver-II. Revista Brasileira de Saúde Materno Infantil, Recife, v. 11, n. 4, p. 445-453, out./dez. 2011. http://dx.doi.org/10.1590/ S1519-38292011000400011

PINTO, E. B.; VILANOVA, L. C. P.; VIEIRA, R. M. $O$ Desenvolvimento do comportamento da criança no primeiro ano de vida. São Paulo: Casa do Psicólogo; FAPESP, 1997. PIPPER, M. C.; DARRAH, J. Motor assessment of the developing infant. Philadelphia: W. B., Saunders Co., 1994.

RANIERO E. P.; TUDELLA, E.; MATTOS, R. S. Padrão e ritmo de aquisição das habilidades motoras de lactentes pré-termo nos quatro primeiros meses de idade corrigida. Revista Brasileira de Fisioterapia, São carlos, v. 14 , n. 5, p. 396-403, set./out. 2010. http://dx.doi. org/10.1590/S1413-35552010000500008

RESEGUE, R.; PUCCINI, R. F.; SILVA, E. M. K. Risk factors associated with developmental abnormalities among high-risk children attended at a multidisciplinary clinic. São Paulo Jornal de Medicina, São Paulo, v. 126, n. 1, p. 4-10, jan. 2008. http://dx.doi.org/10.1590/ S1516-31802008000100002

RESTIFFE, A. P.; GHERPELLI, J. L. Comparison of chronological and corrected ages in the gross motor assessment of low-risk preterm infants during the first year of life. Arquivos de Neuro-Psiquiatria, São Paulo, v. 64, n. 2b, p. 418-425, jun. 2006. http://dx.doi.org/10.1590/ S0004-282X2006000300013

RICHARDSON, P. Use of standardized tests in pediatric practice. In: CASE-SMITH, J. Occupational Therapy for children. St Louis: Mosby, 2001. p. 217-245.

ROSA NETO, F. Manual de Avaliação Motora. Porto Alegre: Artes Médicas, 2002.

SANTOS, R. S.; ARAÚJO, A. P. Q. C.; PORTO, M. A. S. Diagnóstico precoce de anormalidades no desenvolvimento em prematuros: instrumentos de avaliaçáo. Jornal de Pediatria, Rio Janeiro, v. 84, n. 4, p. 289-299, jul./ago. 2008. http://dx.doi.org/10.1590/ S0021-75572008000400003

SILVA, N. D. S. H. et al. Instrumentos de avaliação do desenvolvimento infantil de recém-nascidos prematuros. Revista Brasileira de Crescimento e Desenvolvimento Humano, São Paulo, v. 21, n. 1, p. 85-98, 2011.

TECKLIN, J. S. Fisioterapia Pediátrica. Porto Alegre: Ed. Artmed, 2002.

VIEIRA, V. E. B.; RIBEIRO, F. V.; FORMIGA, C. K. M. R. Principais instrumentos de avaliação do desenvolvimento da criança de zero a dois anos de idade. Movimenta, Goiânia, v. 2, n. 1, p. 23-31, 2009.

WILLIAMS, L. C. A.; AIELLO, A. L. R. O Inventário Portage Operacionalizado: intervenção com famílias. São Paulo: Memnon, 2001. 300 p.

ZANINI, P. Q. et al. Análise da aquisição do sentar, engatinhar e andar em um grupo de crianças pré-termo. Revista de Fisioterapia Universidade de São Paulo, São Paulo, v. 9, n. 2, p. 57-62, jul./dez. 2002.

ZOMIGNANI, A. P.; ZAMBELLI, H. J.; ANTÔNIO, M. A. R. G. M. Desenvolvimento cerebral em recémnascidos prematuros. Revista Paulista de Pediatria, São Paulo, v. 27, n. 2, p. 63-77, jun. 2009. http://dx.doi. org/10.1590/S0103-05822009000200013

\section{Contribuição dos Autores}

Solange Raydan Rocha: concepção do texto, organização de fontes e redação do texto; Lílian de Fátima Dornelas: revisão; Lívia de Castro Magalhães: concepção do texto, redação do texto e revisão. 


\section{Apêndice}

Apêndice 1. Nome e referência bibliográfica dos testes citados.

\begin{tabular}{|c|c|}
\hline $\begin{array}{l}\text { Nome/Sigla do } \\
\text { Instrumento }\end{array}$ & Nome completo e referência \\
\hline AIMS & $\begin{array}{l}\text { Alberta Infant Assessment Scale. PIPER, M. C.; DARRAH, J. Motor assessment of the } \\
\text { developing infant. Philadelphia: W.B, Saunders Co., } 1994 .\end{array}$ \\
\hline BAYLEY II & $\begin{array}{l}\text { Bayley Scales of Infant Development. BAYLEY, N. Bayley Scales of Infant } \\
\text { Development. 2nd ed. San Antonio: The Psychological Corporation, } 1993 .\end{array}$ \\
\hline BRUNET-LEZINE & $\begin{array}{l}\text { Brunet-Lézine's scale. BRUNET, O.; LÉZINE, I. Le dévelopment psychologique de la } \\
\text { premiére enfance. 2nd ed. Paris: Presses Universitaires de France, Italian translation, } \\
1967 .\end{array}$ \\
\hline DENVER II & $\begin{array}{l}\text { Denver Developmental Screening Test. FRANKENBURG, W. K. et al. Denver II } \\
\text { Screening Manual. Denver: Denver Developmental Material, Inc., } 1994 .\end{array}$ \\
\hline DUBOWITZ & $\begin{array}{l}\text { Exame neurológico Dubowitz e Dubowitz. DUBOWITZ, L. M. S.; DUBOWITZ, V.; } \\
\text { GOLDBERG, C. Clinical assesment of gestacional age in the newborn infant. Journal of } \\
\text { Pediatrics, Saint Louis, v. 77, n. 1, p. 1-10, July 1970. http://dx.doi.org/10.1016/S0022- } \\
3476(70) 80038-5\end{array}$ \\
\hline EDCC & $\begin{array}{l}\text { Escala do Comportamento do Desenvolvimento da Criança no } 1^{\circ} \text { ano de vida. PINTO, } \\
\text { E. B.; VILANOVA, L. C. P.; VIEIRA, R. M. O desenvolvimento do comportamento da } \\
\text { criança no primeiro ano de vida. São Paulo: Casa do Psicólogo - FAPESP, } 1997 .\end{array}$ \\
\hline GMFM & $\begin{array}{l}\text { Gross Motor Functional Measure. RUSSEL, D. J. et al. Gross Motor Functional } \\
\text { Measure Manual. 2nd ed. Hamilton: Children's Development Rehabilitation Program, } \\
\text { Hugh MacMillan Rehabilitation Centre, McMaster University, 1993*. }\end{array}$ \\
\hline MAI & $\begin{array}{l}\text { Movement Assessment of Infants. CHANDLER, L. S.; ANDREWS, M. S.; SWANSON, } \\
\text { M. W. Movement Assessment of Infants: A Manual. Washington: University of } \\
\text { Washington, } 1980 .\end{array}$ \\
\hline NAPI & $\begin{array}{l}\text { Neurobehavioral Assessment of the Preterm Infant. KORNER, A. F. et al. The } \\
\text { neurobehavioral assessment of the preterm infant revised. 2nd ed. Van Nuys: Child } \\
\text { Development Media, } 2000 .\end{array}$ \\
\hline Peabody-II & $\begin{array}{l}\text { Peabody Developmental Motor Scales. FOLIO, M. R.; FEWELL, R. R. Peabody } \\
\text { Developmental Motor Scales: Examiner's manual. 2nd ed. (PDMS-2). Austin: Pro-Ed, } \\
2000 .\end{array}$ \\
\hline PEDI & $\begin{array}{l}\text { Pediatric Evaluation of Disability Inventory. MANCINI, M. C. Inventário de Avaliação } \\
\text { Pediátrica de Incapacidade (PEDI): Manual da Versão Brasileira Adaptada. Belo } \\
\text { Horizonte: Ed. UFMG, } 2005 .\end{array}$ \\
\hline
\end{tabular}

*Há uma versão traduzida recente do manual do GMFM (RUSSELL, D. J. et al. Medida da Função Motora Grossa: (GMFM - 66 \& GMFM - 88): Manual do usuário. Tradução de CYRILLO S.T.; GALVÃO M. C. S. São Paulo: Memnon, 2011. 